Keywords: angiogenesis; cytokine; growth factors; inflammation; metastatic colorectal cancer; neutrophil-lymphocyte ratio; survival

\title{
Cytokine profile and prognostic significance of high neutrophil-lymphocyte ratio in colorectal cancer
}

\author{
Z-Y Chen ${ }^{1,9}$, K Raghav ${ }^{2,9}$, C H Lieu ${ }^{3}$, Z-Q Jiang ${ }^{2}, \mathrm{C} \mathrm{Eng}^{2}$, J-N Vauthey ${ }^{4}$, G J Chang ${ }^{4}$, W Qiao ${ }^{5}$, J Morris ${ }^{5}$, \\ D Hong ${ }^{6}$, P Hoff ${ }^{7}, \mathrm{H} \mathrm{Tran}^{8}$, D G Menter ${ }^{2}$, J Heymach ${ }^{8}$, M Overman ${ }^{2}$ and S Kopetz ${ }^{\star 2}$
}

${ }^{1}$ Department of Medical Oncology, Fudan University Shanghai Cancer Center, Shanghai, China; ${ }^{2}$ Department of Gastrointestinal Medical Oncology, The University of Texas MD Anderson Cancer Center, Houston, Texas, USA; ${ }^{3}$ Division of Medical Oncology, University of Colorado, Aurora, Colorado, USA; ${ }^{4}$ Department of Surgical Oncology, The University of Texas MD Anderson Cancer Center, Houston, Texas, USA; ${ }^{5}$ Department of Biostatistics, The University of Texas MD Anderson Cancer Center, Houston, Texas, USA; ${ }^{6}$ Department of Investigational Cancer Therapeutics, The University of Texas MD Anderson Cancer Center, Houston, Texas, USA; Instituto do Cancer do Estado de São Paulo, Faculdade de Medicina da Universidade de São Paulo, Oncologia Clínica, São Paulo, Brazil and ${ }^{8}$ Department of Thoracic/Head \& Neck Medical Oncology, The University of Texas MD Anderson Cancer Center, Houston, Texas, USA

Background: High circulating neutrophil-lymphocyte ratio (NLR) appears to be prognostic in metastatic colorectal cancer (mCRC). We investigated the relationship of NLR with circulating cytokines and molecular alterations.

Methods: We performed retrospective analyses on multiple cohorts of CRC patients (metastatic untreated $(n=166)$, refractory metastatic $(n=161)$, hepatectomy $(n=198)$, stage $2 / 3(n=274)$, and molecularly screened $(n=342))$. High NLR (ratio of absolute neutrophil-to-lymphocyte counts in peripheral blood) was defined as NLR $>5$. Plasma cytokines were evaluated using multiplexbead assays. Kaplan-Meier estimates, non-parametric correlation analysis, and hierarchical cluster analyses were used.

Results: High NLR was associated with poor prognosis in mCRC (hazard ratio (HR) 1.73; 95\% confidence interval (Cl):1.03-2.89; $P=0.039$ ) independent of known prognostic factors and molecular alterations (KRAS/NRAS/BRAF/PIK3CA/CIMP). High NLR correlated with increased expression of interleukin 6 (IL-6), IL-8, IL-2R $\alpha$, hepatocyte growth factor, macrophage-colony stimulating factor, and vascular epidermal growth factor in exploratory $(n=39)$ and validation $(n=166)$ cohorts. Fourteen additional cytokines correlated with high NLR in the validation cohort. All 20 cytokines fell into three major clusters: inflammatory cytokines, angiogenic cytokines, and epidermal growth factor ligands. In mCRC, composite stratification based on NLR-cytokine score provided enhanced prognostic information (HR 2.09; 95\% Cl: 1.59-2.76; $P<0.001$ ) over and above NLR.

Conclusions: High NLR is an independent poor prognostic marker in CRC and correlates with a distinct cytokine profile related to key biological processes involved in carcinogenesis. A composite NLR-cytokine stratification has enhanced prognostic value in $\mathrm{mCRC}$.

Cancer-related inflammation, as either cause or consequence of tumourigenesis, is increasingly recognised as a critical multifaceted player in tumour initiation, growth, and progression
(Mantovani et al, 2008; Colotta et al, 2009). Evidence supports the notion of an inflammatory tumour microenvironment in orchestrating diverse biological processes involved in carcinogenesis

*Correspondence: Dr S Kopetz; E-mail: skopetz@mdanderson.org

${ }^{9} \mathrm{Zhi}$ Yu Chen and Kanwal Raghav are co-first authors of this article.

Received 10 October 2014; revised 29 December 2014; accepted 19 January 2015; published online 17 February 2015

(C) 2015 Cancer Research UK. All rights reserved 0007 - 0920/15 
(Grivennikov et al, 2010). A number of tumour-associated inflammatory cells and cancer-specific cytokines play a pivotal effector role in pro-oncogenic biology (Colotta et al, 2009; Lippitz, 2013). Consequently, extensive research interest is focused on defining and targeting the molecular mechanisms underlying the complex interplay between cancer and inflammation (Coussens et al, 2013).

Cancer-related inflammation appears to be a hallmark of CRC as evidenced by the increased risk of CRC in the setting of chronic bowel inflammation (Ekbom et al, 1990). Additionally, immune scores derived from intratumoural immune infiltrates have strong prognostic impact in CRC (Mlecnik et al, 2011). The driving role of inflammation in CRC is further supported by the reduction in the incidence of CRC and death from CRC in patients taking antiinflammatory drugs (Rothwell et al, 2010). Genomic instability and DNA damage induced by chronic inflammation helps to promote colorectal carcinogenesis (Meira et al, 2008; Yan et al, 2009). Tumour-associated macrophages and several cytokines (e.g., interleukin 6 (IL-6), IL-8) are also implicated in the initiation and progression of CRC (Rubie et al, 2007; Knupfer and Preiss, 2010; Erreni et al, 2011). Cancer-related inflammation and associated systemic inflammatory response have therefore emerged as critical elements governing clinical behaviour of CRC. Multiple inflammatory markers, such as C-reactive protein, and fibrinogen have demonstrated prognostic value in patients with CRC (Koike et al, 2008; Son et al, 2013). Cumulative inflammation-based prognostic scores such as the modified Glasgow prognostic score, based on the levels of C-reactive protein and albumin have been validated in CRC patients undergoing multimodality therapy (Inoue et al, 2013).

The circulating neutrophil-lymphocyte ratio (NLR) is derived from the absolute neutrophil and lymphocyte count obtained from routine complete blood count with differential. Neutrophillymphocyte ratio reflects a systemic inflammatory response and is a readily available and a reliable prognostic marker in multiple tumour types, including CRC (Absenger et al, 2013; Guthrie et al, 2013a; Paramanathan et al, 2014). Elevated NLR has been associated with adverse clinicopathological factors and poor survival outcomes in CRC (Guthrie et al, 2013a; Paramanathan et al, 2014). Furthermore, normalisation of NLR after chemotherapy prior to resection predicts better survival after hepatectomy indicating a predictive role of this marker (Kishi et al, 2009). Clinically, however, the utility of NLR is restricted because of the variable cut-offs used and the less than robust consistent prognostic impact seen in these heterogeneous studies (Guthrie et al, 2013a). It has also been proposed that combining multiple inflammatory markers could result in an incremental improvement in the prognostic value of existent inflammation-based scoring systems (Proctor et al, 2013).

Very little is known about the underlying biology of this subset of patients presenting with an elevated NLR. Circulating cytokines through complex regulatory effects play a key role in determining tumour biology by modulating tumour microenvironment. Emerging data have shown association between cytokines in the inflammatory tumour microenvironment, elevated NLR, and poor survival outcomes (Motomura et al, 2013). Evidence has also revealed that complex cytokine alterations accompanying CRC associate with markers of systemic inflammatory response (Kantola et al, 2012; Lippitz, 2013).

On the basis of these considerations, we performed retrospective analyses to validate the prognostic significance and cut-off of elevated NLR in different clinical settings of CRC. The intent was to evaluate the plausible pathophysiology of this biomarker by identifying the cytokine profile characteristic of this subset of CRC patients. Furthermore, we evaluated the prognostic value of a composite NLR-cytokine stratification in the prediction of overall survival (OS) of patients with metastatic CRC (mCRC).

\section{MATERIALS AND METHODS}

Study population and data collection. We performed retrospective analyses of 1180 patients with CRC who were evaluated and treated at The University of Texas MD Anderson Cancer Center in Houston, Texas. Tumour and blood samples were collected under institutional review board-approved protocols. All patients were divided into six distinct cohorts. Cohort 1 (hereafter referred to as exploratory cohort) consisted of previously untreated mCRC patients $(n=39)$ recruited between January 2005 and January 2007, and treated in a phase II study (Kopetz et al, 2010); this group was used as an exploratory cohort. Cohort 2 (hereafter referred to as validation cohort), included 166 patients with previously untreated $\mathrm{mCRC}$, recruited from April 2004 to December 2008; this group was used as the validation cohort. Cohorts $3(n=161)$ (hereafter referred to as refractory cohort) and $4 \quad(n=198)$ (hereafter referred to as liver-resection cohort) comprised mCRC patients whose cancers were refractory to standard therapies (participants of phase 1 clinical trials: April 2005 to December 2009) or who had resected liver-limited disease (received perioperative chemotherapy: August 2002 to February 2006), respectively. Cohort $5(n=274)$ (hereafter referred to as stage II/III cohort) included patients with resected stage II/III disease recruited from July 2000 to November 2008. Cohort 6 (hereafter referred to as ATTACC cohort) included an additional 342 with treatment refractory mCRC patients enrolled on the ATTACC (Assessment of Targeted Therapies Against Colorectal Cancer) (Supplementary Data) protocol, and was screened for KRAS, NRAS, BRAF, PIK3CA mutations, PTEN loss, and CpG island methylation (CIMP) between June 2010 and June 2013; it used to evaluate the association of NLR and molecular alterations. Clinical demographics and baseline characteristics, including age, sex, performance status, TNM stage, tumour location, lactate dehydrogenase level, complete blood count with differential, and survival time from diagnosis, were collected using the MD Anderson Cancer Center CRC database and chart review.

Neutrophil-lymphocyte ratio. Neutrophil-lymphocyte ratios were calculated retrospectively from the ratio of peripheral blood absolute neutrophil and lymphocyte counts at baseline. For cohorts 1, 2, 4, and 5, NLRs were calculated using counts at or near diagnosis prior to initiation of therapy. For cohort 3, NLRs were computed from counts prior to phase I study enrolment. Using a previously determined cut-off from other studies, patients were segregated into those with a low $\operatorname{NLR}(\leqslant 5)$ and those with a high NLR (>5) (Kishi et al, 2009). Additionally, we performed a separate analysis to determine whether 5 was the optimal cut-off to use for NLR. For this, area under the curve for HRs and 95\% confidence intervals (CIs) corresponding to different NLR values were compared in the validation cohort.

Plasma sample collection and analysis. Cytokine profiling to determine plasma cytokine levels encompassed the exploratory cohort (cohort 1) and validation cohort (cohort 2) patients. Plasma cytokine levels were assessed using multiplex bead assay (Bio-Rad Laboratories, Hercules, CA, USA and EMD, Bioscience Research Reagents, Temecula, CA, USA) as previously described (Kopetz et al, 2010). Epidermal growth factor assays were incorporated to evaluate the interaction of NLR with EGFR ligand levels. Plasma samples were drawn and collected in ethylenediaminetetraacetic acid tubes and centrifuged at 2800 r.p.m. for $10 \mathrm{~min}$ at $-22^{\circ} \mathrm{C}$. Sample aliquots were placed into $0.5-\mathrm{ml}$ cryovials and stored at $-70{ }^{\circ} \mathrm{C}$ to $-80^{\circ} \mathrm{C}$ until analysis. Plasma samples (in 500- $\mu$ l aliquots) were thawed in parallel and each used for suspension bead multiplex assays. Levels of 42 (exploratory cohort) and 51 (validation cohort) cytokines were measured by suspension multiplex bead assays and were analyzed per the 
manufacturers' directions. Cytokine concentrations from all samples were analyzed in the same multiplex bead suspension arrays in triplicate to minimise interexperimental variability. For each individual sample, the analysis was repeated if the coefficient of variance was $<25 \%$. Plasma samples from 15 patients without cancer from the validation cohort were run as controls. Please note that we have all circulating markers that were assessed are collectively referred to as cytokines for simplicity but include chemokines, growth factors, soluble receptors, and others.

Composite NLR-cytokine stratification. Cytokines that significantly correlated with NLR were selected and a cytokine score (CS) was calculated as the sum of the Z-scores of this given cytokine set for individual patients (individual Z-score was calculated as the difference of the average and individual cytokine level divided by standard deviation of $\log 2$-transformed value). Using the CS, patients were dichotomised into those with high CS and low CS using the median value as cut-off. A composite stratification was then created using NLR and CS. All patients were categorised by the combination of NLR and CS into three groups: a high NLR-CS group (high CS and NLR), an intermediate NLR-CS group (high CS with low NLR or low CS with high NLR), and a low NLR-CS group (low CS and low NLR).

Statistical methods. The primary endpoint of the study was to compare the OS between the high NLR and low NLR group of patients with mCRC. The OS and corresponding censoring were computed in months from diagnosis to death for each patient. Additionally, disease-free survival was computed for liver-resection patients and was defined as the interval between hepatectomy and recurrent disease. Median OS, 3-year OS, and disease-free survival were estimated non-parametrically using the Kaplan-Meier method and compared by the log-rank test. Cox proportional hazards and linear regression model was performed to identify prognostic factors in univariate and multivariate analysis. The Fisher's exact test was used to determine the association between NLR and molecular alterations. The Wilcoxon rank-sum test was used to estimate the differences in cytokine expression between the two NLR groups. Owing to variable cytokine levels, NLR and cytokine expression levels were log2-transformed to render the data set symmetric. Subsequently, supervised hierarchical cluster analysis was performed to determine clusters between NLR and cytokines with significant differential expression. Correlations between NLR and cytokines were assessed by Pearson analysis and set the order for cytokines in a coherence heatmap plot on the basis of orders of clusters identified in the hierarchical cluster analysis.

A $P$-value less than 0.05 was considered statistically significant in two-sided non-parametric tests. For multiple comparisons in the correlation analyses of cytokines and NLR, we adjusted the $P$-value boundary using the Bonferroni method to control for experimentwise error rate.

\section{RESULTS}

Baseline characteristics. The median age at presentation was 56.9 years (range, 26-79 years). Table 1 summarises the baseline patient characteristics, which were, overall, well-balanced between the high and low NLR groups in both the exploratory and the validation cohorts. Patients with high NLR were more likely to have lactate dehydrogenase levels above the upper limit of normal (75\% vs 33\%; $P=0.035)$, lower performance status $(75 \%$ vs $37 \% ; P=0.040)$ and leukocyte counts greater than $11000 \mathrm{ml}^{-1}$ (42\% vs $4 \%$; $\left.P=0.007\right)$ in the exploratory cohort. Similar differences were also seen in the validation cohorts.
Neutrophil-lymphocyte ratio and molecular alterations. Among the 342 patients in cohort 6, KRAS, NRAS, BRAF, PIK3CA mutations, PTEN loss, and CIMP testing was successfully performed on 342 (100\%), 201 (59\%), 281 (82\%), 229 (67\%), 272 (79\%), and 310 (90\%) patients, respectively. In the screened cases, KRAS, NRAS, BRAF, PIK3CA mutation, PTEN loss, and CIMPhigh phenotype was seen in $52.6 \%, 7.5 \%, 7.5 \%, 19.2 \%, 11.8 \%$, and $21.6 \%$ cases, respectively. There was no statistically significant association between NLR and mutations in KRAS (odds ratio (OR) 0.98; 95\% CI: 0.57-1.69; $P=1.000$ ), NRAS (OR: 0.64; 95\% CI: $0.14-2.97 ; \quad P=0.741$ ), BRAF (OR: $0.70 ; 95 \%$ CI: $0.19-2.47$; $P=0.775$ ), or PIK3CA (OR: 0.95 ; 95\% CI: $0.41-2.23$; $P=1.000$ ). Similarly, there was no association of NLR with PTEN loss (OR: 1.06; 95\% CI: $0.41-2.73$; $P=1.000$ ) or CIMP (OR: 1.46 ; 95\% CI: 0.76-2.79; $\mathrm{P}=0.291$ ) (Figure 1).

Neutrophil-lymphocyte ratio validation as a prognostic factor in diverse CRC cohorts and cut-off optimisation. In the analysis to corroborate the optimal cut off value of NLR, comparison among the multiple NLR cut-offs confirmed that the previously reported cut-off value of 5 was associated with the most optimal HR (Supplementary Figure 1).

In all settings of mCRC, patients with high NLR had worse median OS than patients with low NLR, as seen in the validation cohort (15.3 months vs 34.2 months) and refractory cohort (3.7 months vs 9.0 months). This difference in median OS was statistically significant in both groups, with HR of 2.74 (95\% CI: $1.75-4.30 ; P<0.001)$ and 2.15 (95\% CI: $1.49-3.11 ; P<0.001)$ in validation and refractory cohorts, respectively. Similarly, median disease-free survival and 3-year OS were lower in patients with high NLR (9.5 months and 8\%) than in those with low NLR (13.3 months and 38\%) in liver-resection cohort (HR 1.83; 95\% CI: 1.142.93; $P=0.010)$. The 3 -year OS was worse in patients with high NLR (75\%) than in those with low NLR (90\%) in stage II/III cohort (HR 2.37; 95\% CI: $1.1-5.1 ; P=0.023$ ). These survival estimates and Kaplan-Meier curves are shown in Figure 2 (Supplementary Table 1). In further exploratory analyses of individual components of NLR, that is, neutrophil count and lymphocyte count, we found that high neutrophil counts alone provided most of the prognostic information contained in the NLR but that absolute lymphocyte levels were also prognostic and/or added to the prognostic ability of the neutrophil count in most of the cohorts (Supplementary Figure 2 and 3).

Table 2 summarises the results of the univariate and multivariate analyses that were performed on patients in cohort 2 . Multiple metastatic sites $(\geqslant 1)$, elevated lactate dehydrogenase $(\geqslant$ upper limit of normal), elevated total leukocyte count $\left(\geqslant 11000 \mu \mathrm{l}^{-1}\right)$, poor performance status, and high NLR were predictive of worse OS in univariate analysis. On multivariate analysis, multiple metastatic sites and elevated lactate dehydrogenase continued to be significantly associated with poor OS. High NLR was found to be independently associated with a higher risk of death, after adjustment for other variables (HR 1.73; 95\% CI: 1.03-2.89; $P=0.039$ ).

Differential cytokine expression in exploratory and validation cohorts. Table 3 summarises the results of cytokine analysis in exploratory and validation cohorts. Six cytokines were expressed at significantly higher levels in the high NLR group than in the low NLR group in the exploratory cohort. These exploratory cohort cytokines included (IL-6, IL-8, IL-2R $\alpha$, hepatocyte growth factor (HGF), macrophage-colony stimulating factor (or colony stimulating factor 1 receptor, M-CSF or CSF-1), and vascular epidermal growth factor A (VEGF-A)). These results were validated in the validation cohort.

An additional 13 cytokines were found to be significantly elevated in the high NLR group in the validation cohort (chemokine (C-X-C motif) ligand 1 (CXCL1 or GRO $\alpha$ ); IL-18; 
Table 1. Baseline patient characteristics in exploratory and validation cohorts

\begin{tabular}{|c|c|c|c|c|c|c|c|c|}
\hline \multirow[b]{2}{*}{ Variables } & \multicolumn{4}{|c|}{ Exploratory cohort (cohort 1) } & \multicolumn{4}{|c|}{ Validation cohort (cohort 2) } \\
\hline & $\begin{array}{l}\text { Total } \\
N(\%)\end{array}$ & $\begin{array}{l}\text { NLR } \leqslant 5 \\
N(\%)\end{array}$ & $\begin{array}{c}\text { NLR }>5 \\
N(\%)\end{array}$ & $\begin{array}{l}\text { Fisher } \\
P \text {-value }\end{array}$ & $\begin{array}{l}\text { Total } \\
N(\%)\end{array}$ & $\begin{array}{c}\text { NLR } \leqslant 5 \\
N(\%)\end{array}$ & $\begin{array}{c}\text { NLR }>5 \\
N(\%)\end{array}$ & $\begin{array}{l}\text { Fisher } \\
P \text {-value }\end{array}$ \\
\hline All patients $(N)$ & 39 & 27 & 12 & - & 166 & 126 & 40 & - \\
\hline Median age (years) (range) & $56.8(26-78)$ & $56(34-75)$ & $58(26-78)$ & - & $57(30-79)$ & $56.5(30-79)$ & $58(30-72)$ & - \\
\hline \multicolumn{9}{|l|}{ Sex } \\
\hline $\begin{array}{l}\text { Male } \\
\text { Female }\end{array}$ & $\begin{array}{l}24(62) \\
15(38)\end{array}$ & $\begin{array}{l}16(60) \\
11(40)\end{array}$ & $\begin{array}{l}8(67) \\
4(33)\end{array}$ & 0.730 & $\begin{array}{l}96(58) \\
70(42)\end{array}$ & $\begin{array}{l}73(58) \\
53(42)\end{array}$ & $\begin{array}{l}23(58) \\
17(42)\end{array}$ & 0.960 \\
\hline \multicolumn{9}{|l|}{ Race/ethnicity } \\
\hline $\begin{array}{l}\text { White } \\
\text { Black } \\
\text { Hispanic } \\
\text { Others }\end{array}$ & $\begin{aligned} 32 & (82) \\
2 & (5) \\
5 & (13) \\
& -\end{aligned}$ & $\begin{aligned} & 21(78) \\
& 2(7) \\
& 4(15) \\
&-\end{aligned}$ & $\begin{array}{l}11(92) \\
0(0) \\
1(8) \\
-\end{array}$ & 1.000 & $\begin{array}{c}124(75) \\
20(12) \\
16(10) \\
6(3)\end{array}$ & $\begin{array}{c}96(76) \\
12(10) \\
14(11) \\
4(3)\end{array}$ & $\begin{aligned} & 28(70) \\
& 8(20) \\
& 2(5) \\
& 2(5)\end{aligned}$ & 0.200 \\
\hline \multicolumn{9}{|c|}{ ECOG performance status } \\
\hline $\begin{array}{l}0 \\
1 \\
2 \\
3\end{array}$ & $\begin{array}{c}20(51) \\
19(49) \\
- \\
-\end{array}$ & $\begin{array}{c}17(63) \\
10(37) \\
- \\
-\end{array}$ & $\begin{array}{r}3(25) \\
9(75) \\
- \\
-\end{array}$ & 0.040 & $\begin{array}{l}58(35) \\
74(45) \\
24(14) \\
10(6)\end{array}$ & $\begin{array}{l}48(38) \\
55(44) \\
19(15) \\
4(3)\end{array}$ & $\begin{aligned} 10(25) \\
19(47) \\
5(13) \\
6(15)\end{aligned}$ & 0.045 \\
\hline \multicolumn{9}{|l|}{ Primary site } \\
\hline $\begin{array}{l}\text { Colon } \\
\text { Recto-sigmoid } \\
\text { Rectum }\end{array}$ & $\begin{array}{r}28(72) \\
4(10) \\
7(18)\end{array}$ & $\begin{aligned} 20 & (74) \\
1 & (4) \\
6 & (22)\end{aligned}$ & $\begin{array}{l}8(67) \\
3(25) \\
1(8)\end{array}$ & 0.140 & $\begin{array}{r}108(65) \\
28(17) \\
30(18)\end{array}$ & $\begin{array}{l}79(63) \\
22(17) \\
25(20)\end{array}$ & $\begin{array}{r}29(72) \\
6(15) \\
5(13)\end{array}$ & 0.480 \\
\hline \multicolumn{9}{|c|}{ Metastatic sites (number) } \\
\hline $\begin{array}{l}<1 \\
\geqslant 1\end{array}$ & $\begin{array}{r}9(23) \\
30(77)\end{array}$ & $\begin{array}{r}8(30) \\
19(70)\end{array}$ & $\begin{array}{c}1(8) \\
11(92)\end{array}$ & 0.230 & $\begin{array}{l}90(54) \\
76(46)\end{array}$ & $\begin{array}{r}72(57) \\
54(43)\end{array}$ & $\begin{array}{l}18(45) \\
22(55)\end{array}$ & 0.180 \\
\hline \multicolumn{9}{|l|}{ Leukocyte count } \\
\hline $\begin{array}{l}<11000 \mu \mathrm{l}^{-1} \\
\geqslant 11000 \mu \mathrm{l}^{-1}\end{array}$ & $\begin{array}{r}33(85) \\
6(15)\end{array}$ & $\begin{array}{l}26(96) \\
1(4)\end{array}$ & $\begin{array}{l}7(58) \\
5(42)\end{array}$ & 0.007 & $\begin{array}{r}132(80) \\
34(20)\end{array}$ & $\begin{array}{r}114(90) \\
12(10)\end{array}$ & $\begin{array}{l}18(45) \\
22(55)\end{array}$ & $<0.01$ \\
\hline \multicolumn{9}{|l|}{ Lactate dehydrogenase } \\
\hline $\begin{array}{l}<\text { Upper limit of normal } \\
\geqslant \text { Upper limit of normal }\end{array}$ & $\begin{array}{l}21(54) \\
18(46)\end{array}$ & $\begin{array}{r}18(67) \\
9(33)\end{array}$ & $\begin{array}{l}3(25) \\
9(75)\end{array}$ & 0.035 & $\begin{array}{l}92(55) \\
71(43)\end{array}$ & $\begin{array}{l}83(66) \\
40(32)\end{array}$ & $\begin{array}{r}9(23) \\
31(77)\end{array}$ & $<0.01$ \\
\hline
\end{tabular}

IL-12; macrophage migration inhibitory factor (MIF); chemokine (C-C motif) ligand 4 or macrophage inflammatory protein- $1 \beta$ (CCL4 or MIP-1 $\beta$ ); C-type lectin domain family 11 , member A or stem cell growth factor- $\beta$ (CLEC11A or SCGF- $\beta$ ) and EGF; transforming growth factor- $\alpha$ (TGF- $\alpha$ ); heparin-binding EGF-like growth factor (HB-EGF); amphiregulin (AREG); epiregulin (EREG); intercellular adhesion molecule-1 (ICAM-1); and tenascin-C). The only cytokine expressed at significantly higher levels in the low NLR group compared with the high NLR group was TNFrelated apoptosis-inducing ligand (TRAIL), also known as CD253 or tumour necrosis factor superfamily, member 10 (TNFSF10). The proportional change in the 20 differentially expressed cytokines is shown in Supplementary Figure 4. Nineteen of the 20 cytokines with the exception of TRAIL showed high expression in patients with high NLR.

Supervised hierarchical clustering analysis of cytokines and correlation with NLR. The subset of 20 cytokines helped identify clusters that reflect the tumour biology underpinnings of elevated NLR. Three major clusters were generated by supervised hierarchical clustering analyses and a coherence matrix representing separate functional orders as follows: angiogenic cytokines (IL6 , IL-8, IL-12, and VEGF-A), pro-inflammatory cytokines (GRO $\alpha$, IL-2R $\alpha$, HGF, MIF, IL-18, and SCGF- $\beta$ ) and epidermal growth factor ligands (AREG, EGF, HB-EGF, EREG, and TGF- $\alpha$ ). The expression heatmap and coherence heatmap based on the coefficient matrix of the Spearman correlation are shown in Supplementary Figure 5.

We then calculated the statistical correlations between the 20 cytokine panel and NLR using Spearman correlation analyses ( $P$-value adjusted for multiple comparisons). Eleven cytokines (HGF,
IL-6, IL-8, IL-18, IL-2R $\alpha$, GRO $\alpha$, ICAM-1, SCGF- $\beta$, MIP-1 $\beta$, tenascin- $C$, and AREG) exhibited positive correlations with high NLR that were highly significant $(P \leqslant 0.001)$. TRAIL showed a negative correlation with high NLR that was highly significant $(P \leqslant 0.001$; Supplementary Tables 2 and 3$)$.

Composite NLR-cytokine stratification. To assess the prognostic impact of cytokine profile and the incremental benefit in addition to the NLR, we evaluated the prognostic implication of the CS and the composite NLR-cytokine stratification (Figure 3). Dichotomised CS (cut-off: median value of CS) was prognostic for OS, and patients having high CS showed worse median OS (17.2 months) than patients with low scores (40.0 months) (HR 2.29; 95\% CI: $1.51-3.48 ; P<0.001)$. The combined NLR-CS-based stratification segregated the cohort into three distinct prognostic groups, with median OS of 40.2 months, 26.4 months, and 8.9 months in the low-, intermediate-, and high-risk groups, respectively (HR 2.09; 95\% CI: $1.59-2.76 ; P<0.001)$. These OS data indicate the prognostic value of NLR-CS-based stratification.

\section{DISCUSSION}

Cancer-related inflammation is a pro-oncogenic influence resulting from an intricate tumour-host interface that is progressively being recognised as a hallmark of carcinogenesis (Mantovani et al, 2008; Colotta et al, 2009). This localised and systemic inflammatory milieu is a crucial determinant of tumour biology (Mantovani et al, 2008; Grivennikov et al, 2010). Multiple inflammatory markers are emerging as surrogate biomarkers for this cancer-related systemic inflammatory response, and prognostic indicators in CRC patients 

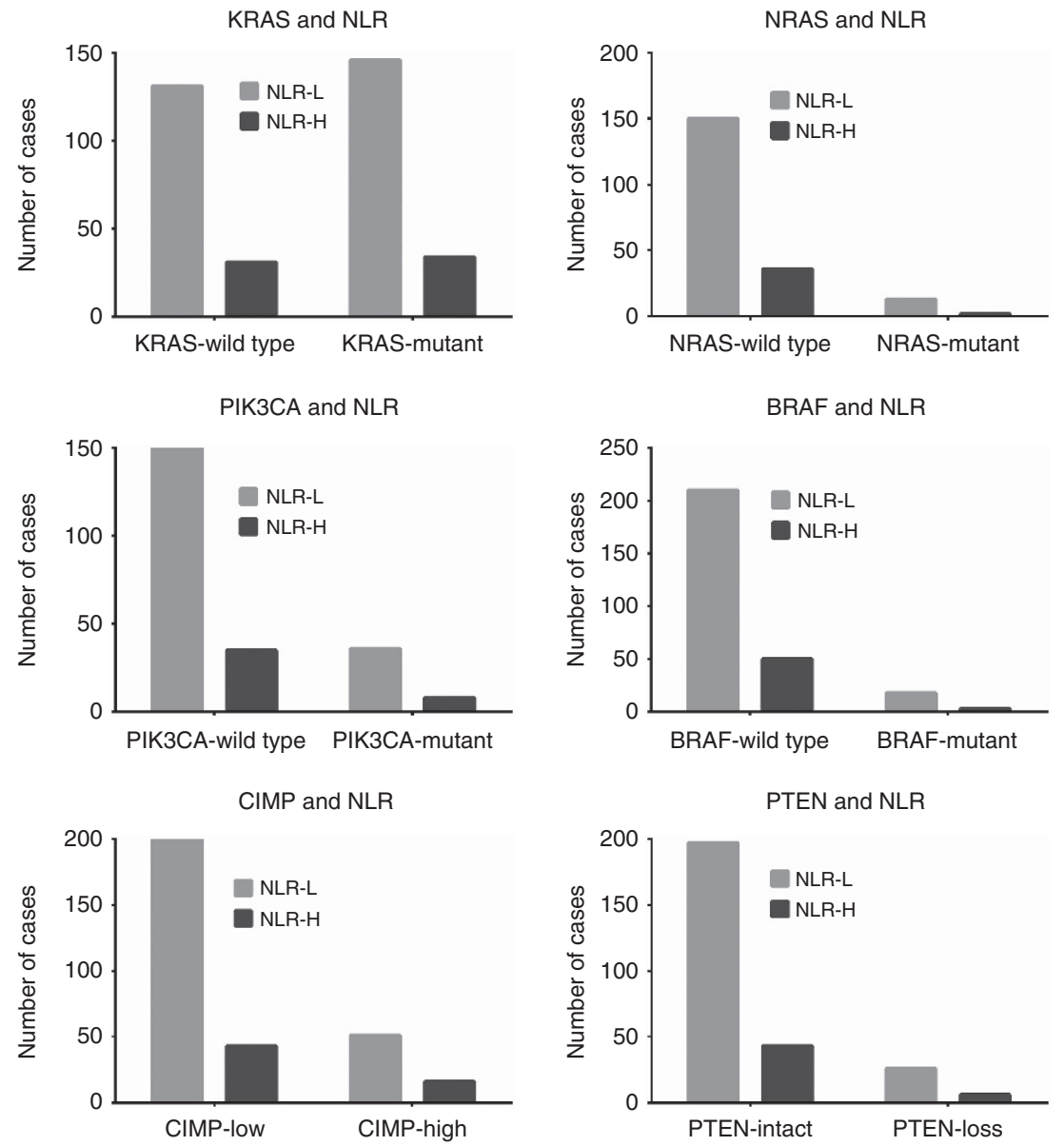

Figure 1. Bar-graphs showing association of neutrophil-lymphocyte ratio (NLR) with molecular alterations (mutations in KRAS, NRAS, BRAF, PIK3CA; PTEN loss, and CpG island methylator phenotype (CIMP) in metastatic colorectal cancer. High NLR is defined as a NLR $>5$.

(Koike et al, 2008; Son et al, 2013; Li et al, 2014). The circulating NLR, defined as the ratio of the total peripheral blood neutrophil count to the total peripheral blood lymphocyte count, is one such marker which has been evaluated in multiple cancers, including CRC, and has shown prognostic significance (Guthrie et al, 2013a; Li et al, 2014).

Our analysis of patients with CRC demonstrates that high NLR is a reliable prognostic factor across multiple settings of CRC. Specifically these settings include, surgically resected stage II/III CRC (15\% lower 3-year OS rate), mCRC with liver metastasis after hepatectomy (30\% lower 3-year OS rate), and previously untreated and refractory mCRC (55\% and 59\% lower median OS times, respectively). We have also identified the optimal cut-off value of $\operatorname{NLR}(>5$ and $\leqslant 5)$ in mCRC. We demonstrated here that NLR is not associated with any of the common molecular alterations seen in mCRC, including KRAS, NRAS, BRAF, PIK3CA mutations, PTEN loss, and CIMP testing. In addition, to the best of our knowledge, this study is the first to establish a more comprehensive expression signature of circulating cytokines in mCRC patients with an elevated NLR. Furthermore, we demonstrated that combining CS with NLR greatly enhanced prognostic stratification in mCRC. Understanding the subtleties of these combined clinical measures is expected to better elucidate disease progression.

Systemic inflammatory markers (e.g., C-reactive protein) and inflammation-based scores (e.g., modified Glasgow prognostic score) are gaining acceptance as prognostic indicators in CRC (Koike et al, 2008; Inoue et al, 2013). Neutrophil-lymphocyte ratio, as the surrogate indicator of this systemic inflammatory response has prognostic significance in CRC; however, its cut-off and validity across multiple settings of CRC has been uncertain
(Guthrie et al, 2013a; Li et al, 2014). A meta-analyses of studies from various countries confirmed the prognostic value of NLR in CRC (Li et al, 2014). Four of 10 studies of surgically resectable CRC and 2 of 2 studies of advanced CRC characterised NLR as being independently associated with survival ( $\mathrm{Li}$ et al, 2014). However, these studies are heterogeneous and used varying cutoffs for elevated NLR (Guthrie et al, 2013a; Li et al, 2014). The current study is among the largest to evaluate the prognostic impact of elevated NLR in multiple settings of CRC. These findings establish that elevated NLR portends poor prognosis in CRC and determined NLR $>5$ as a statistically optimal cut-off value for NLR associated with the highest degree of discrimination of outcomes. Interestingly, the prognostic value of NLR also persists in heavily pretreated patients, despite the potential impact of prior chemotherapy on bone marrow function. In addition, as suggested by recent studies, we found that in $\mathrm{mCRC}$, majority of prognostic information of NLR is derived from neutrophil count (Proctor et al, 2012). Additionally, we also found that the relative prognostic information of either absolute neutrophil or lymphocyte counts may differ by setting and/or stage of the disease. We propose that NLR is a reliable biomarker of cancer-related inflammation and clinical behaviour that can be used for risk stratification in CRC for treatment, surveillance, and prognosis. Elevated NLR appears to identify a group of CRC patients that have an atypical biology; however, the mechanisms underlying this phenotype remain poorly understood.

$\mathrm{CRC}$ is recognised as a heterogeneous disease resulting from progressive accumulation of genetic and epigenetic alteration (Cancer Genome Atlas N, 2012). These molecular alterations appear to play a key role in tumour biology and behaviour. 

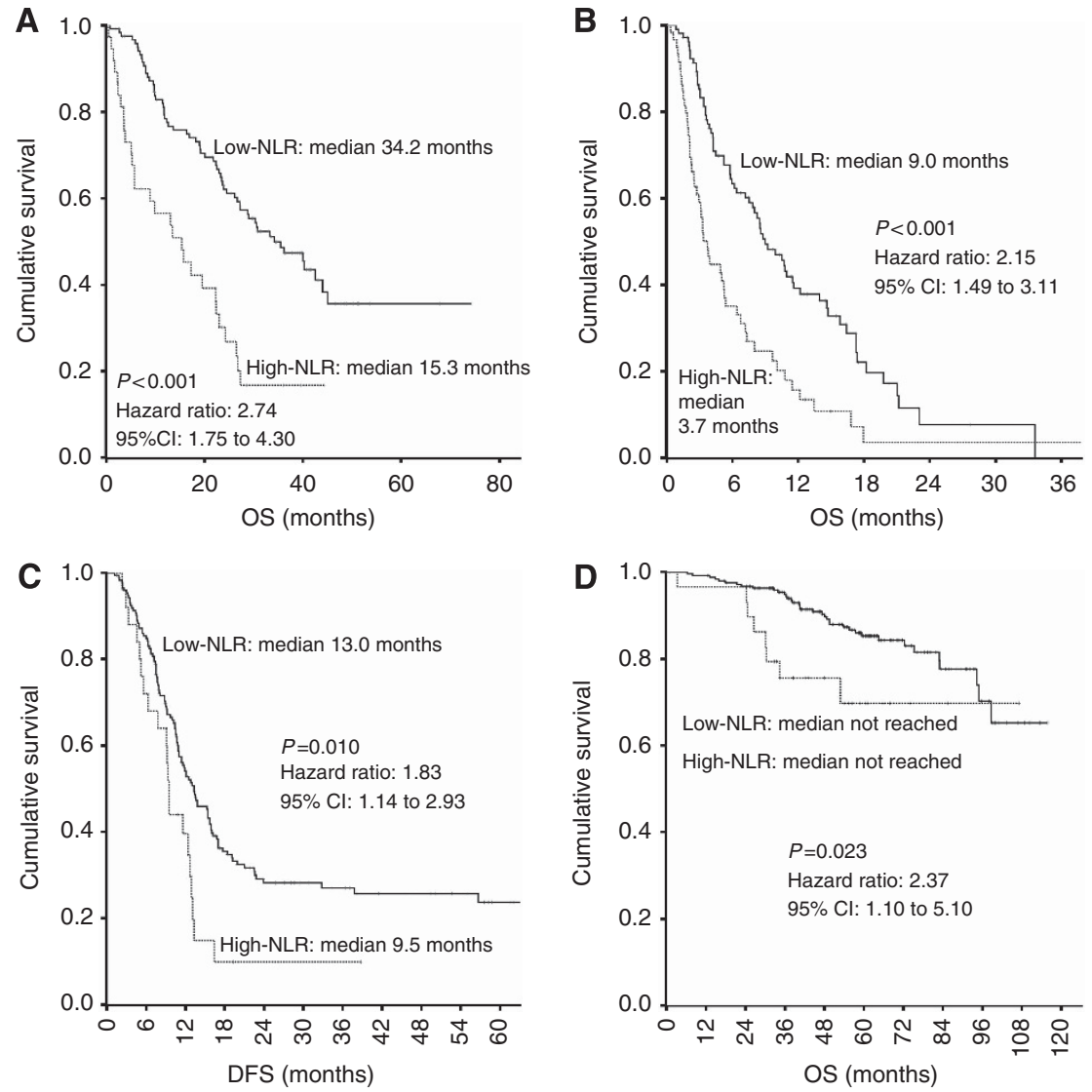

Figure 2. Kaplan-Meier survival curves illustrating overall survival (OS) and disease-free survival (DFS) in different settings of colorectal cancer (CRC), compared between patients with a high neutrophil-lymphocyte ratio (NLR), defined as NLR $>5$, and those with a low NLR (NLR $\leqslant 5$ ). (A) OS in cohort 2 (previously untreated metastatic CRC). (B) OS in cohort 3 (refractory metastatic CRC). (C) Disease-free survival in cohort 4 (liver metastatic disease after hepatectomy). (D) OS in cohort 5 (stage II/III CRC after surgery).

Consequently, classification based on molecular subtypes of CRC has gained popularity for prognostication and treatment. We investigated and found no association of NLR with common molecular alterations seen in CRC suggesting that the high NLR phenotype and its aggressive biology is independent of some molecular subtypes of CRC including KRAS, NRAS, BRAF, PIK3CA mutations, PTEN loss, and CIMP.

Cytokines regulate multiple biological processes involved in carcinogenesis, including inflammation (Mantovani et al, 2008). Upregulated pro-oncogenic cytokines such as IL-6 exhibit prognostic utility in CRC among other cancers (Kantola et al, 2012; Lippitz, 2013). We revealed that 20 cytokines are differentially expressed in the high NLR subset of mCRC. We showed that IL-6 was significantly upregulated in patients with high NLR. When observed in the circulation, IL-6 is linked to tumour necrosis, systemic and local inflammatory responses in patients undergoing resection for colorectal cancer (Guthrie et al, 2013b). Both, the preoperative serum IL-6 levels and granulocyte/lymphocyte ratio appear to be clinically relevant biomarkers of long-term cancer progression (Shimazaki et al, 2013). This body of work suggests that IL-6 may function dynamically as tumours evolve. It is also consistent with the behaviour of our larger panel of CS.

It is conceivable that the various cytokine clusters act dynamically along with IL-6 as functional drivers of systemic inflammatory response and tumour evolution and may be linked to markers of inflammation, specifically NLR, in CRC patients. This is the first study of its kind to explore the relationship between elevated NLR and cytokines in patients with mCRC. We identified 19 cytokines using supervised clustering analysis with high expression levels in mCRC patients with high NLR. On the basis of clustering, the cytokines identified are functionally involved in angiogenesis, inflammation, and tumour growth promotion. Functional overlap occurs between these clusters as shown in Figure 2. For example, IL-6, a pro-inflammatory cytokine is also involved in angiogenesis. Most of these cytokines (IL-6, IL-8, HGF, GRO $\alpha$, IL-2R $\alpha$, M-CSF, and VEGF-A) were individually observed in prior studies to associate with disease progression, poor survival, and poor treatment outcomes in patients with mCRC, indicating the pivotal role of these cytokines in tumour biology (Lissoni et al, 1990; Wen et al, 2006; Mroczko et al, 2007; Rubie et al, 2007; Knupfer and Preiss, 2010; Kopetz et al, 2010; Jurgensmeier et al, 2013). Notably, five EGFR ligands (EGF, AREG, EREG, TGF- $\alpha$, and HB-EGF) were consistently upregulated in our patients with elevated NLR. In prior studies, elevated EGFR ligands have shown to have prognostic and predictive effects on survival in CRC, many of which concur with our findings, including AREG, HB-EGF, TGF-alpha, and EREG (Ohchi et al, 2012; Pentheroudakis et al, 2013; Yoshida et al, 2013). In the present study, AREG levels were 61 -fold higher along with 163-fold higher TGF- $\alpha$ level in high NLR patients. Another molecule that increased in our high NLR subsets was tenascin-C, an extracellular matrix glycoprotein with 14 EGFlike structural repeats. Tenascin-C has been shown to associate with poor prognosis in CRC (Emoto et al, 2001). Collectively, elevated circulating EGFR ligand findings in conjunction with high NLR suggest that the EGFR signalling pathway is associated with the pro-inflammatory CRC phenotype.

Supervised clustering analysis not only revealed circulating proinflammatory cytokines and EGFR ligands but also pro-angiogenic factors in high NLR patients. For instance, GRO $\alpha$, encoded by the CXCL1 gene and also known as neutrophil-activating protein 3 
Table 2. Univariate and multivariate analyses of clinical prognostic factors for overall survival in 166 patients with mCRC

\begin{tabular}{|c|c|c|c|c|c|c|c|c|c|}
\hline \multirow[b]{2}{*}{ Variables } & \multirow[b]{2}{*}{$N$} & \multirow[b]{2}{*}{$\begin{array}{l}\text { Median OS } \\
\text { (months) }\end{array}$} & \multirow[b]{2}{*}{$95 \% \mathrm{Cl}$} & \multicolumn{3}{|c|}{ Univariate analysis } & \multicolumn{3}{|c|}{ Multivariate analysis } \\
\hline & & & & HR & $95 \% \mathrm{Cl}$ & $P$ & HR & $95 \% \mathrm{Cl}$ & $P$ \\
\hline \multicolumn{10}{|l|}{ Age (years) } \\
\hline $\begin{array}{l}\leqslant 65 \\
>65\end{array}$ & $\begin{array}{r}114 \\
52\end{array}$ & $\begin{array}{l}28.7 \\
24.5\end{array}$ & $\begin{array}{l}18.5-38.9 \\
20.4-28.5\end{array}$ & 1.19 & $0.76-1.86$ & 0.450 & & & \\
\hline \multicolumn{10}{|l|}{ Sex } \\
\hline $\begin{array}{l}\text { Male } \\
\text { Female }\end{array}$ & $\begin{array}{l}96 \\
70\end{array}$ & $\begin{array}{l}28.7 \\
26.4\end{array}$ & $\begin{array}{l}20.0-32.6 \\
21.8-31.1\end{array}$ & 0.89 & $0.59-1.34$ & 0.570 & & & \\
\hline \multicolumn{10}{|l|}{ Primary site } \\
\hline $\begin{array}{l}\text { Colon } \\
\text { Recto-sigmoid } \\
\text { Rectum }\end{array}$ & $\begin{array}{r}108 \\
28 \\
30\end{array}$ & $\begin{array}{l}26.1 \\
35.4 \\
28.7\end{array}$ & $\begin{array}{l}22.3-29.9 \\
24.4-46.5 \\
10.6-46.9\end{array}$ & 1.00 & $0.77-1.31$ & 0.960 & & & \\
\hline \multicolumn{10}{|c|}{ Leukocyte count } \\
\hline $\begin{array}{l}<11000 \mathrm{ml}^{-1} \\
\geqslant 11000 \mathrm{ml}^{-1}\end{array}$ & $\begin{array}{r}133 \\
33\end{array}$ & $\begin{array}{l}33.2 \\
13.1\end{array}$ & $\begin{array}{c}22.8-43.7 \\
3.9-22.2\end{array}$ & 2.46 & $1.53-3.93$ & $<0.01$ & 0.80 & $0.42-1.53$ & 0.497 \\
\hline \multicolumn{10}{|l|}{ ECOG PS } \\
\hline $\begin{array}{l}0 \\
\geqslant 1\end{array}$ & $\begin{array}{r}58 \\
108\end{array}$ & $\begin{array}{l}42.5 \\
22.3\end{array}$ & $\begin{array}{l}37.8-47.1 \\
18.4-26.2\end{array}$ & 2.27 & $1.39-3.69$ & 0.001 & 1.39 & $0.88-2.19$ & 0.158 \\
\hline \multicolumn{10}{|c|}{ Metastatic sites (number) } \\
\hline $\begin{array}{l}<1 \\
\geqslant 1\end{array}$ & $\begin{array}{l}90 \\
76\end{array}$ & $\begin{array}{l}\text { NA } \\
16.9\end{array}$ & $\begin{array}{c}\text { NA } \\
21.8-32.6\end{array}$ & 3.48 & $2.23-5.40$ & $<0.01$ & 2.94 & $1.87-4.63$ & $<0.01$ \\
\hline \multicolumn{10}{|l|}{ LDH } \\
\hline $\begin{array}{l}<U L N \\
\geqslant U L N\end{array}$ & $\begin{array}{l}92 \\
71\end{array}$ & $\begin{array}{l}40.2 \\
15.7\end{array}$ & $\begin{array}{c}29.9-50.6 \\
8.5-22.8\end{array}$ & 2.65 & $1.73-4.05$ & $<0.01$ & 1.86 & $1.14-3.03$ & 0.012 \\
\hline \multicolumn{10}{|l|}{ NLR } \\
\hline $\begin{array}{l}\leqslant 5 \\
>5\end{array}$ & $\begin{array}{r}126 \\
40\end{array}$ & $\begin{array}{l}34.2 \\
15.3\end{array}$ & $\begin{array}{c}25.1-43.3 \\
6.9-23.7\end{array}$ & 2.74 & $1.75-4.30$ & $<0.01$ & 1.73 & $1.03-2.89$ & 0.039 \\
\hline
\end{tabular}

(NAP-3), produced by colorectal tumour cells or leukocytes in the tumour microenvironment acts as an autocrine growth factor and promotes tumour progression and angiogenesis (Wang et al, 2006; Galamb et al, 2012). Similarly, pro-angiogenic factors (IL-6, IL-8, IL-12, and VEGF-A) are also important for neutrophil recruitment, T-cell differentiation, and tumour growth (Erreni et al, 2011). All these cytokines were found to be elevated in the high NLR subtype of mCRC.

Decreases in programmed cell death also influence tumour growth. TRAIL was the only cytokine that was significantly downregulated in patients with elevated NLR. TRAIL is the death ligand of the TNF family and mediates caspase-8-dependent apoptosis in malignant cells (Van Geelen et al, 2004). This deficiency in the apoptotic signalling pathway in patients with elevated NLR may explain the tumours' aggressive biology.

We developed a composite stratification that included NLR and a CS derived from 12 high NLR-associated cytokines to refine the prognostic value of combining these circulating markers. The CS derived from these cytokines was also found to be prognostic and patients with high CS had shorter survival than patients with low CS. In order to determine whether NLR recapitulated the prognostic information in the cytokine profile, we evaluated the incremental benefit of a CS over NLR-based stratification. When examined, the combined composite NLR-CS stratification showed that patients with elevated NLR and elevated CS had the poorest prognosis. This composite stratification enhanced the prognostic value of elevated NLR, indicating that although prognostic, NLR does not fully reflect the entire prognostic information available from the cytokine profile.

The ability to improve risk stratification of CRC patients allows us to investigate novel therapeutic targets for this subset of CRC patients. Similar analyses have revealed plasma-derived predictive markers in pancreatic cancer (Nixon et al, 2013). Future studies are needed to validate the predictive nature of this stratification allowing for incorporation of these inflammatory markers to guide cancer therapy. For example, AREG and EREG have been shown to predict cetuximab efficacy in CRC and consequently, elevated NLR patients with elevated AREG levels may be more likely to benefit from anti-EGFR therapy (Pentheroudakis et al, 2013). This is a hypothesis that requires testing in randomised studies of antiEGFR therapy. Similarly, low TRAIL levels following bevacizumab therapy portend poor survival, and low TRAIL levels in elevated NLR may be a marker for bevacizumab resistance in mCRC patients (Bisgin et al, 2012). Other opportunities exist for neutralising cancer-promoting inflammation as a promising new strategy for anti-cancer therapies (Coussens et al, 2013). For instance, the downregulation of GRO $\alpha$ can inhibit tumour growth in CRC liver metastasis (Bandapalli et al, 2012). Humanised antibodies to IL-8 (ABX-IL8), which have been shown to inhibit melanoma tumour growth, angiogenesis, and metastasis, may have a potential clinical benefit in CRC with elevated NLR (Zigler et al, 2008). Likewise, TRAIL-directed therapies may show more clinical benefit in patients with elevated NLR (Stolfi et al, 2012).

The authors are aware that because the current study is a retrospective analysis, it may suffer from some unavoidable biases. Many co-morbidities and conditions such as infections and drugs can influence NLR restricting its utility as a predictive or prognostic biomarker. The retrospective nature of this study limits our ability to adjust for effects of these variables. To this effect, efforts are needed to incorporate NLR prospectively as a prognostic biomarker in clinical studies. Although not all differentially expressed cytokines were validated in both exploratory and validation cohorts, we believe that the small numbers in the exploratory cohort could be responsible for the lack of consistent 
Table 3. Differential expression of cytokines comparing high NLR and low NLR groups in exploratory (cohort 1) and validation (cohort 2) cohorts

\begin{tabular}{|c|c|c|c|c|c|c|}
\hline \multirow[b]{3}{*}{ Cytokines $^{a}$} & \multicolumn{3}{|c|}{ Exploratory cohort (cohort 1) $(N=39)$} & \multicolumn{3}{|c|}{ Validation cohort (cohort 2) $(N=166)$} \\
\hline & \multicolumn{3}{|c|}{ Median } & \multicolumn{3}{|c|}{ Median } \\
\hline & Low NLR & High NLR & $P$-value & Low NLR & High NLR & $P$-value \\
\hline IL-8 & 3.060 & 10.95 & 0.021 & 19.44 & 50.00 & $<0.001$ \\
\hline $\mathrm{IL}-2 \mathrm{R} \alpha$ & 179.7 & 235.5 & 0.006 & 183.8 & 268.2 & $<0.001$ \\
\hline VEGF-A & 32.73 & 118.5 & 0.015 & 13.51 & 29.01 & 0.004 \\
\hline IL-12 & 390.5 & 440.1 & 0.498 & 8.820 & 18.72 & 0.010 \\
\hline MIP-1 $\beta$ & 36.54 & 34.31 & 0.855 & 59.76 & 77.43 & $<0.001$ \\
\hline $\mathrm{GRO} \alpha$ & 104.7 & 132.3 & 0.222 & 2.030 & 49.85 & $<0.001$ \\
\hline Amphiregulin & & $\mathrm{NE}$ & & 3.120 & 189.4 & $<0.001$ \\
\hline EGF & & $\mathrm{NE}$ & & 11.47 & 28.24 & 0.016 \\
\hline Epiregulin & & $\mathrm{NE}$ & & 4.770 & 24.40 & 0.038 \\
\hline HB-EGF & & $\mathrm{NE}$ & & 87.05 & 203.3 & 0.001 \\
\hline Tenascin & & $\mathrm{NE}$ & & 1118 & 1602 & 0.001 \\
\hline TGF- $\alpha$ & & $\mathrm{NE}$ & & 0.030 & 4.090 & 0.025 \\
\hline ICAM-1 & & $\mathrm{NE}$ & & 124061 & 189463 & 0.001 \\
\hline \multicolumn{7}{|c|}{ 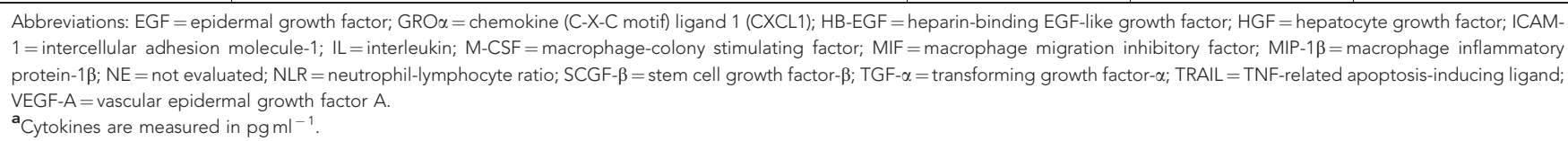 } \\
\hline
\end{tabular}
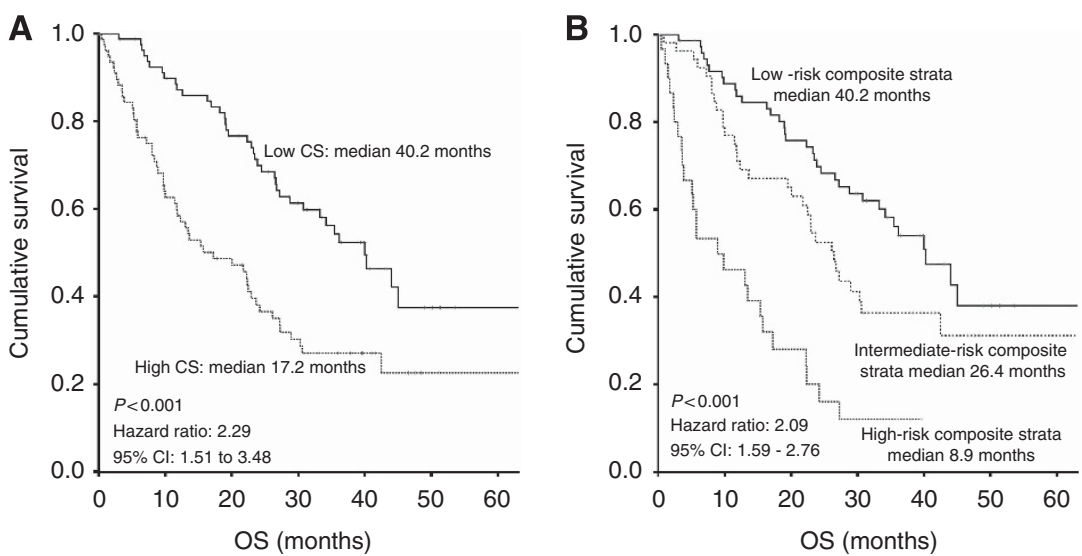

Figure 3. Kaplan-Meier survival curves illustrating OS in patients with metastatic colorectal cancer (CRC) using (A) CS and (B) Composite NLR-CS stratification.

effect for some of the cytokines. Six cytokines were expressed at significantly higher levels in the high NLR group than in the low NLR group in the exploratory cohort. These exploratory cohort cytokines included (IL-6, IL-8, IL-2R $\alpha$, HGF, macrophage-colony stimulating factor (or colony stimulating factor 1 receptor, M-CSF or CSF-1R) and vascular epidermal growth factor A (VEGF-A)). These results were validated in the validation cohort. The additional 13 cytokines were found to be significantly elevated in the high NLR group in the validation cohort only. Although our data show a strong correlation between high NLR and elevated cytokines, whether this high NLR-associated cytokine profile is a reflection, a determinant, or a contributor of tumour biology in this subset of CRC patients is yet to be determined. These findings represent the systemic response to cancer and may not fully reflect the local tumour microenvironment, which was not assessed in this study. Furthermore, although we have been able to establish association of NLR with these cytokines, causality cannot be established from this analysis. Mechanistic studies are necessary to define the role of these cytokines in this subset. Although we have found several expression clusters of cytokines in high NLR 
patients, we recognise that many more cytokines may play integrated roles in the biology of this subset of mCRCs. As C-reactive protein is not assessed routinely in patients with $\mathrm{mCRC}$, we had limited ability to assess the association of this cytokine profile with the Glasgow prognostic score, and future research is needed.(Proctor et al, 2013)

Evidence implicating local and systemic inflammatory response in progression of tumours and survival of cancer patients suggests that inflammation is a key player in cancer biology and targeting these inflammatory responses can potentially improve patient outcomes (Guthrie et al, 2013a; Diakos et al, 2014). Recent data have identified NLR as a robust prognostic indicator in early phase clinical trials suggesting its potential for selecting patients for experimental therapeutics (Pinato et al, 2014). Neutrophillymphocyte ratio has also been shown to predict prognosis for cancer patients undergoing curative resection and may potentially identify subsets of patients who may benefit from adjuvant chemotherapy.(Paramanathan et al, 2014) Prospective validation of composite stratifications such as the NLR-CS will be instrumental in further improving this risk stratification in colorectal cancer.

\section{CONCLUSIONS}

In conclusion, NLR is an inexpensive, readily available, and reliable biomarker for the prediction of survival in patients with CRC. CRC patients with elevated NLR $(>5)$ have tumours characterised by aggressive biology and a distinctive expression profile of cytokines involved in angiogenesis, inflammation, and regulation of the EGF axis. An integrated NLR-CS is a novel inflammation-based prognostic stratification with enhanced predictive value for survival in patients with mCRC. Neutrophil-lymphocyte ratio and composite NLR-CS can potentially be used as clinical stratification tools to identify a unique biological subset of CRCs. Future trials should be designed toward targeting this unique inflammatory state in CRC.

\section{REFERENCES}

Absenger G, Szkandera J, Stotz M, Postlmayr U, Pichler M, Ress AL, Schaberl-Moser R, Loibner H, Samonigg H, Gerger A (2013) Preoperative neutrophil-to-lymphocyte ratio predicts clinical outcome in patients with stage II and III colon cancer. Anticancer Res 33(10): 4591-4594.

Bandapalli OR, Ehrmann F, Ehemann V, Gaida M, Macher-Goeppinger S, Wente M, Schirmacher P, Brand K (2012) Down-regulation of CXCL1 inhibits tumor growth in colorectal liver metastasis. Cytokine 57(1): 46-53.

Bisgin A, Kargi A, Yalcin AD, Aydin C, Ekinci D, Savas B, Sanlioglu S (2012) Increased serum sTRAIL levels were correlated with survival in bevacizumab-treated metastatic colon cancer. BMC Cancer 12: 58.

Cancer Genome Atlas N (2012) Comprehensive molecular characterization of human colon and rectal cancer. Nature 487(7407): 330-337.

Colotta F, Allavena P, Sica A, Garlanda C, Mantovani A (2009) Cancer-related inflammation, the seventh hallmark of cancer: links to genetic instability. Carcinogenesis 30(7): 1073-1081.

Coussens LM, Zitvogel L, Palucka AK (2013) Neutralizing tumor-promoting chronic inflammation: a magic bullet? Science 339(6117): 286-291.

Diakos CI, Charles KA, McMillan DC, Clarke SJ (2014) Cancer-related inflammation and treatment effectiveness. Lancet Oncol 15(11): e493-e503.

Ekbom A, Helmick C, Zack M, Adami HO (1990) Ulcerative colitis and colorectal cancer. A population-based study. N Engl J Med 323(18): $1228-1233$

Emoto K, Yamada Y, Sawada H, Fujimoto H, Ueno M, Takayama T, Kamada K, Naito A, Hirao S, Nakajima Y (2001) Annexin II overexpression correlates with stromal tenascin-C overexpression: a prognostic marker in colorectal carcinoma. Cancer 92(6): 1419-1426.
Erreni M, Mantovani A, Allavena P (2011) Tumor-associated Macrophages (TAM) and Inflammation in Colorectal Cancer. Cancer Microenviron 4(2): 141-154

Galamb O, Wichmann B, Sipos F, Spisak S, Krenacs T, Toth K, Leiszter K, Kalmar A, Tulassay Z, Molnar B (2012) Dysplasia-carcinoma transition specific transcripts in colonic biopsy samples. PLoS One 7(11): e48547.

Grivennikov SI, Greten FR, Karin M (2010) Immunity, inflammation, and cancer. Cell 140(6): 883-899.

Guthrie GJ, Charles KA, Roxburgh CS, Horgan PG, McMillan DC, Clarke SJ (2013a) The systemic inflammation-based neutrophil-lymphocyte ratio: Experience in patients with cancer. Crit Rev Oncol Hematol 88(1): 218-230.

Guthrie GJ, Roxburgh CS, Richards CH, Horgan PG, McMillan DC (2013b) Circulating IL-6 concentrations link tumour necrosis and systemic and local inflammatory responses in patients undergoing resection for colorectal cancer. Br J Cancer 109(1): 131-137.

Inoue Y, Iwata T, Okugawa Y, Kawamoto A, Hiro J, Toiyama Y, Tanaka K, Uchida K, Mohri Y, Miki C, Kusunoki M (2013) Prognostic significance of a systemic inflammatory response in patients undergoing multimodality therapy for advanced colorectal cancer. Oncology 84(2): 100-107.

Jurgensmeier JM, Schmoll HJ, Robertson JD, Brooks L, Taboada M, Morgan SR, Wilson D, Hoff PM (2013) Prognostic and predictive value of VEGF, sVEGFR-2 and CEA in mCRC studies comparing cediranib, bevacizumab and chemotherapy. Br J Cancer 108(6): 1316-1323.

Kantola T, Klintrup K, Vayrynen JP, Vornanen J, Bloigu R, Karhu T, Herzig KH, Napankangas J, Makela J, Karttunen TJ, Tuomisto A, Makinen MJ (2012) Stage-dependent alterations of the serum cytokine pattern in colorectal carcinoma. Br J Cancer 107(10): 1729-1736.

Kishi Y, Kopetz S, Chun YS, Palavecino M, Abdalla EK, Vauthey IN (2009) Blood neutrophil-to-lymphocyte ratio predicts survival in patients with colorectal liver metastases treated with systemic chemotherapy. Ann Surg Oncol 16(3): 614-622.

Knupfer H, Preiss R (2010) Serum interleukin-6 levels in colorectal cancer patients-a summary of published results. Int J Colorectal Dis 25(2): 135-140.

Koike Y, Miki C, Okugawa Y, Yokoe T, Toiyama Y, Tanaka K, Inoue Y, Kusunoki M (2008) Preoperative C-reactive protein as a prognostic and therapeutic marker for colorectal cancer. J Surg Oncol 98(7): 540-544.

Kopetz S, Hoff PM, Morris JS, Wolff RA, Eng C, Glover KY, Adinin R, Overman MJ, Valero V, Wen S, Lieu C, Yan S, Tran HT, Ellis LM, Abbruzzese JL, Heymach JV (2010) Phase II trial of infusional fluorouracil, irinotecan, and bevacizumab for metastatic colorectal cancer: efficacy and circulating angiogenic biomarkers associated with therapeutic resistance. J Clin Oncol 28(3): 453-459.

Li MX, Liu XM, Zhang XF, Zhang JF, Wang WL, Zhu Y, Dong J, Cheng JW, Liu ZW, Ma L, Lv Y (2014) Prognostic role of neutrophil-to-lymphocyte ratio in colorectal cancer: a systematic review and meta-analysis. Int J Cancer 134(10): 2403-2413.

Lippitz BE (2013) Cytokine patterns in patients with cancer: a systematic review. Lancet Oncol 14(6): e218-e228.

Lissoni P, Barni S, Rovelli F, Viviani S, Maestroni GJ, Conti A, Tancini G (1990) The biological significance of soluble interleukin-2 receptors in solid tumors. Eur J Cancer 26(1): 33-36.

Mantovani A, Allavena P, Sica A, Balkwill F (2008) Cancer-related inflammation. Nature 454(7203): 436-444.

Meira LB, Bugni JM, Green SL, Lee CW, Pang B, Borenshtein D, Rickman BH Rogers AB, Moroski-Erkul CA, McFaline JL, Schauer DB, Dedon PC, Fox JG, Samson LD (2008) DNA damage induced by chronic inflammation contributes to colon carcinogenesis in mice. J Clin Invest 118(7): 2516-2525.

Mlecnik B, Tosolini M, Kirilovsky A, Berger A, Bindea G, Meatchi T, Bruneval P, Trajanoski Z, Fridman WH, Pages F, Galon J (2011) Histopathologicbased prognostic factors of colorectal cancers are associated with the state of the local immune reaction. J Clin Oncol 29(6): 610-618.

Motomura T, Shirabe K, Mano Y, Muto J, Toshima T, Umemoto Y, Fukuhara T, Uchiyama H, Ikegami T, Yoshizumi T, Soejima Y, Maehara Y (2013) Neutrophil-lymphocyte ratio reflects hepatocellular carcinoma recurrence after liver transplantation via inflammatory microenvironment. $J$ Hepatol 58(1): 58-64.

Mroczko B, Groblewska M, Wereszczynska-Siemiatkowska U, Okulczyk B, Kedra B, Laszewicz W, Dabrowski A, Szmitkowski M (2007) Serum macrophage-colony stimulating factor levels in colorectal cancer patients correlate with lymph node metastasis and poor prognosis. Clin Chim Acta 380(1-2): 208-212.

Nixon AB, Pang H, Starr MD, Friedman PN, Bertagnolli MM, Kindler HL, Goldberg RM, Venook AP, Hurwitz HI. Alliance for Clinical Trials In O 
(2013) Prognostic and predictive blood-based biomarkers in patients with advanced pancreatic cancer: results from CALGB80303 (Alliance). Clin Cancer Res 19(24): 6957-6966.

Ohchi T, Akagi Y, Kinugasa T, Kakuma T, Kawahara A, Sasatomi T, Gotanda Y, Yamaguchi K, Tanaka N, Ishibashi Y, Miyamoto S, Kage M, Shirouzu K (2012) Amphiregulin is a prognostic factor in colorectal cancer. Anticancer Res 32(6): 2315-2321.

Paramanathan A, Saxena A, Morris DL (2014) A systematic review and meta-analysis on the impact of pre-operative neutrophil lymphocyte ratio on long term outcomes after curative intent resection of solid tumours. Surg Oncol 23(1): 31-39.

Pentheroudakis G, Kotoula V, De Roock W, Kouvatseas G, Papakostas P, Makatsoris T, Papamichael D, Xanthakis I, Sgouros J, Televantou D, Kafiri G, Tsamandas AC, Razis E, Galani E, Bafaloukos D, Efstratiou I, Bompolaki I, Pectasides D, Pavlidis N, Tejpar S, Fountzilas G (2013) Biomarkers of benefit from cetuximab-based therapy in metastatic colorectal cancer: interaction of EGFR ligand expression with RAS/RAF, PIK3CA genotypes. BMC Cancer 13: 49.

Pinato DJ, Stavraka C, Flynn MJ, Forster MD, O’Cathail SM, Seckl MJ, Kristeleit RS, Olmos D, Turnbull SJ, Blagden SP (2014) An inflammation based score can optimize the selection of patients with advanced cancer considered for early phase clinical trials. PLoS One 9(1): e83279.

Proctor MJ, Horgan PG, Talwar D, Fletcher CD, Morrison DS, McMillan DC (2013) Optimization of the systemic inflammation-based Glasgow Prognostic Score: A Glasgow Inflammation Outcome Study. Cancer 119(12): 2325-2332.

Proctor MJ, McMillan DC, Morrison DS, Fletcher CD, Horgan PG, Clarke SJ (2012) A derived neutrophil to lymphocyte ratio predicts survival in patients with cancer. Br J Cancer 107(4): 695-699.

Rothwell PM, Wilson M, Elwin CE, Norrving B, Algra A, Warlow CP, Meade TW (2010) Long-term effect of aspirin on colorectal cancer incidence and mortality: 20-year follow-up of five randomised trials. Lancet 376(9754): 1741-1750.

Rubie C, Frick VO, Pfeil S, Wagner M, Kollmar O, Kopp B, Graber S, Rau BM, Schilling MK (2007) Correlation of IL-8 with induction, progression and metastatic potential of colorectal cancer. World J Gastroenterol 13(37): 4996-5002.
Shimazaki J, Goto Y, Nishida K, Tabuchi T, Motohashi G, Ubukata H, Tabuchi T (2013) In patients with colorectal cancer, preoperative serum interleukin-6 level and granulocyte/lymphocyte ratio are clinically relevant biomarkers of long-term cancer progression. Oncology 84(6): 356-361.

Son HJ, Park JW, Chang HJ, Kim DY, Kim BC, Kim SY, Park SC, Choi HS, Oh JH (2013) Preoperative plasma hyperfibrinogenemia is predictive of poor prognosis in patients with nonmetastatic colon cancer. Ann Surg Oncol 20(9): 2908-2913.

Stolfi C, Pallone F, Monteleone G (2012) Molecular targets of TRAILsensitizing agents in colorectal cancer. Int J Mol Sci 13(7): 7886-7901.

Van Geelen CM, de Vries EG, de Jong S (2004) Lessons from TRAILresistance mechanisms in colorectal cancer cells: paving the road to patient-tailored therapy. Drug Resist Updat 7(6): 345-358.

Wang D, Wang H, Brown J, Daikoku T, Ning W, Shi Q, Richmond A, Strieter R, Dey SK, DuBois RN (2006) CXCL1 induced by prostaglandin E2 promotes angiogenesis in colorectal cancer. J Exp Med 203(4): 941-951.

Wen Y, Giardina SF, Hamming D, Greenman J, Zachariah E, Bacolod MD, Liu H, Shia J, Amenta PS, Barany F, Paty P, Gerald W, Notterman D (2006) GROalpha is highly expressed in adenocarcinoma of the colon and down-regulates fibulin-1. Clin Cancer Res 12(20 Pt 1): 5951-5959.

Yan B, Peng Y, Li CY (2009) Molecular analysis of genetic instability caused by chronic inflammation. Methods Mol Biol 512: 15-28.

Yoshida M, Shimura T, Sato M, Ebi M, Nakazawa T, Takeyama H, Joh T (2013) A novel predictive strategy by immunohistochemical analysis of four EGFR ligands in metastatic colorectal cancer treated with anti-EGFR antibodies. J Cancer Res Clin Oncol 139(3): 367-378.

Zigler M, Villares GJ, Lev DC, Melnikova VO, Bar-Eli M (2008) Tumor immunotherapy in melanoma: strategies for overcoming mechanisms of resistance and escape. Am J Clin Dermatol 9(5): 307-311.

This work is published under the standard license to publish agreement. After 12 months the work will become freely available and the license terms will switch to a Creative Commons AttributionNonCommercial-Share Alike 4.0 Unported License.

Supplementary Information accompanies this paper on British Journal of Cancer website (http://www.nature.com/bjc) 